\title{
Fusariosis in haematological malignancy - the skin is the clue... Experiences from the National Cancer Institute of Sri Lanka: A case report
}

MN Jayawardena ${ }^{1}$, SHCK De Silva ${ }^{1}$, NP Madarasingha ${ }^{2}$, M Somathilake ${ }^{3}$, S Wanigasooriya $^{3}$, PR Chinthamani $^{1}$, LSM Sigera ${ }^{4}$, PGRIS Welagedara ${ }^{4}$, KDDS Wijeweera ${ }^{1}$, PGRUM Welagedara ${ }^{1}$, YK Perera $^{1}$, NWES Nugahapola ${ }^{1}$, UE Rathnayake ${ }^{1}$, SP Gunasekera ${ }^{1}$, PI Jayasekera ${ }^{4}$

Sri Lankan Journal of Infectious Diseases 2021Vol.11(1):52-57

DOI: http://dx.doi.org/10.4038/sljid.v11i1.8336

\begin{abstract}
We present two patients with haematological malignancies who developed skin lesions while neutropaenic and were subsequently diagnosed as having fusariosis. Although fusariosis is not as common as other fungal infections such as aspergillosis and candidiasis, it has to be considered in the diagnosis of immunocompromised patients who present with skin manifestations. Awareness of fusariosis, and early diagnosis and appropriate treatment is essential to reduce mortality.
\end{abstract}

Keywords: Fusarium, Sri Lanka, haematological malignancies

\section{Introduction}

The Fusarium species is a mold that can cause invasive and disseminated infections in severely immunocompromised patients, such as patients with prolonged or profound neutropaenia and Tcell mediated immunodeficiencies. Skin manifestations of this infection are common in these patients. We present two children with leukemia who presented with dermatological manifestations of fusariosis.

We emphasize that fungal infections should be considered in the differential diagnosis of those with neutropaenia and dermatological manifestations. The two case studies presented here

\footnotetext{
${ }^{1}$ Department of Microbiology, National Cancer Institute, Maharagama, Sri Lanka

${ }^{2}$ Department of Dermatology, National Cancer Institute, Maharagama, Sri Lanka

${ }^{3}$ Department of Paediatric Oncology, National Cancer Institute, Maharagama, Sri Lanka

${ }^{4}$ Department of Mycology, Medical Research Institute, Colombo 8, Sri Lanka

Address for correspondence: Dr MN Jayawardena. Department of Microbiology, National Cancer Institute, Maharagama, Sri Lanka. Telephone: +94773074599/+447852 356110. E-mail: naamaljay@gmail.com

https://orcid.org/0000-0003-1928-8086

Received 10 December 2020 and revised version accepted 8 March 2021.

(c) (7) This an open-access article distributed under the terms of the Creative Commons Attribution License, which permits unrestricted use, distribution, and reproduction in any medium, provided the original author and source are credited.
} 
highlight that early diagnosis and initiation of antifungal therapy is essential for a favourable outcome.

\section{Patient 1}

A 11-year old boy was admitted to the paediatric oncology ward for chemotherapy following a relapse of acute lymphoblastic leukaemia (ALL). The patient was started on a very high-risk induction with vincristine, daunorubicin and cyclophosphamide. He complained of headache and gum-bleeding on admission and was pale but afebrile. On day 3, he developed a fever of $100{ }^{\circ} \mathrm{F}$. He was pancytopaenic [absolute neutrophil count (ANC) $210 / \mu \mathrm{L}$, with a haemoglobin of $8.8 \mathrm{~g} / \mathrm{dL}$, platelets $\left.14 \times 10^{3} / \mu \mathrm{L}\right]$ and had oral thrush. After taking blood and urine cultures, the patient was started on IV meropenem $20 \mathrm{mg} / \mathrm{kg}$ 8hourly and IV amikacin $15 \mathrm{mg} / \mathrm{kg} 12$ hourly. After 15 hours of incubation, the blood culture signaled positive for Staphylococcus aureus, while his urine culture remained sterile. In response, IV flucloxacillin 500mg 6hourly was started.

IV fluconazole $12 \mathrm{mg} / \mathrm{kg}$ daily was added as the patient continued to be neutropaenic (ANC $20 / \mu \mathrm{L}$ ) and had fever spikes after an initial positive response to antibiotics. . As the patient remained afebrile, his chemotherapy regime was continued.

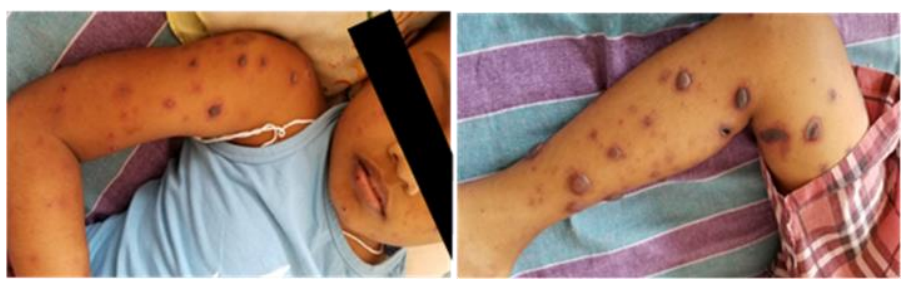

Figure 1: Skin rash of the patient taken two days before expiry, showing erythematous macules, targetoid lesions with central necrosis and haemorrhagic bullae
Two weeks after his admission, the fever recurred, and the patient developed a skin rash. This started with erythematous macules, turning into targetoid lesions with central necrosis (Figure 1). His lesions were tender and situated more toward the extremities. Some of the lesions turned into bullae, which later became haemorrhagic. After seeking the dermatologist's opinion, the patient was suspected to have either a drug reaction or a disseminated fungal infection. Therefore, an aspirate from the bullae was taken for bacterial and fungal cultures. However, a skin biopsy was not performed due to low platelet counts.

After taking blood for culture and omitting flucloxacillin and IV fluconazole, the patient was started on IV clindamycin $12 \mathrm{mg} / \mathrm{kg}$ 6hourly, IV vancomycin $15 \mathrm{mg} / \mathrm{kg}$ 8hourly and IV amphotericin B (liposomal) 3mg/kg daily. The aspirate yielded a Fusarium species (direct smear and culture positive), identified at the Mycology Reference Laboratory (Figure 2). Further speciation was difficult due to the unavailability of molecular diagnostic methods. Based on the epidemiological cut-off values provided by $\mathrm{CLSI}^{1}$ the minimum inhibitory concentrations amphotericin B and voriconazole for this isolate were $0.19 \mu \mathrm{g} / \mathrm{l}$ and more than $32 \mu \mathrm{g} / \mathrm{l}$ respectively. 
The blood cultures signaled positive for two types of coliforms and streptococcal species, which were sensitive to meropenem and vancomycin / clindamycin respectively. Meropenem was converted to Meronem ${ }^{\mathrm{TM}}$ $500 \mathrm{mg}$ 8hourly and the vancomycin and clindamycin continued. Sadly, the patient expired two days later. He was only given five days of amphotericin B for his fungal skin infection.

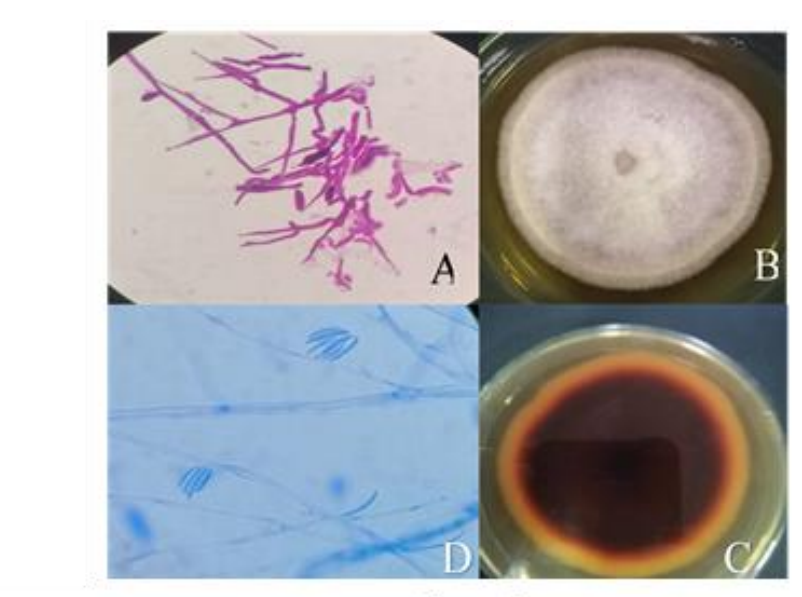

Figure 2:

A: Gram stain

B: Culture morphology of isolate, on SDA after one week incubation at $37^{\circ} \mathrm{C}$,(obverse) showing rapidly growing floccose white colonies with aerial mycelia and $\mathrm{C}$ : vinaceous red (reverse)

D: Tease mount on lactophenol cotton blue, prepared from the culture showing abundant macroconidia with 3-5 septa and absent chlamydospores.

\section{Patient 1- Timeline}

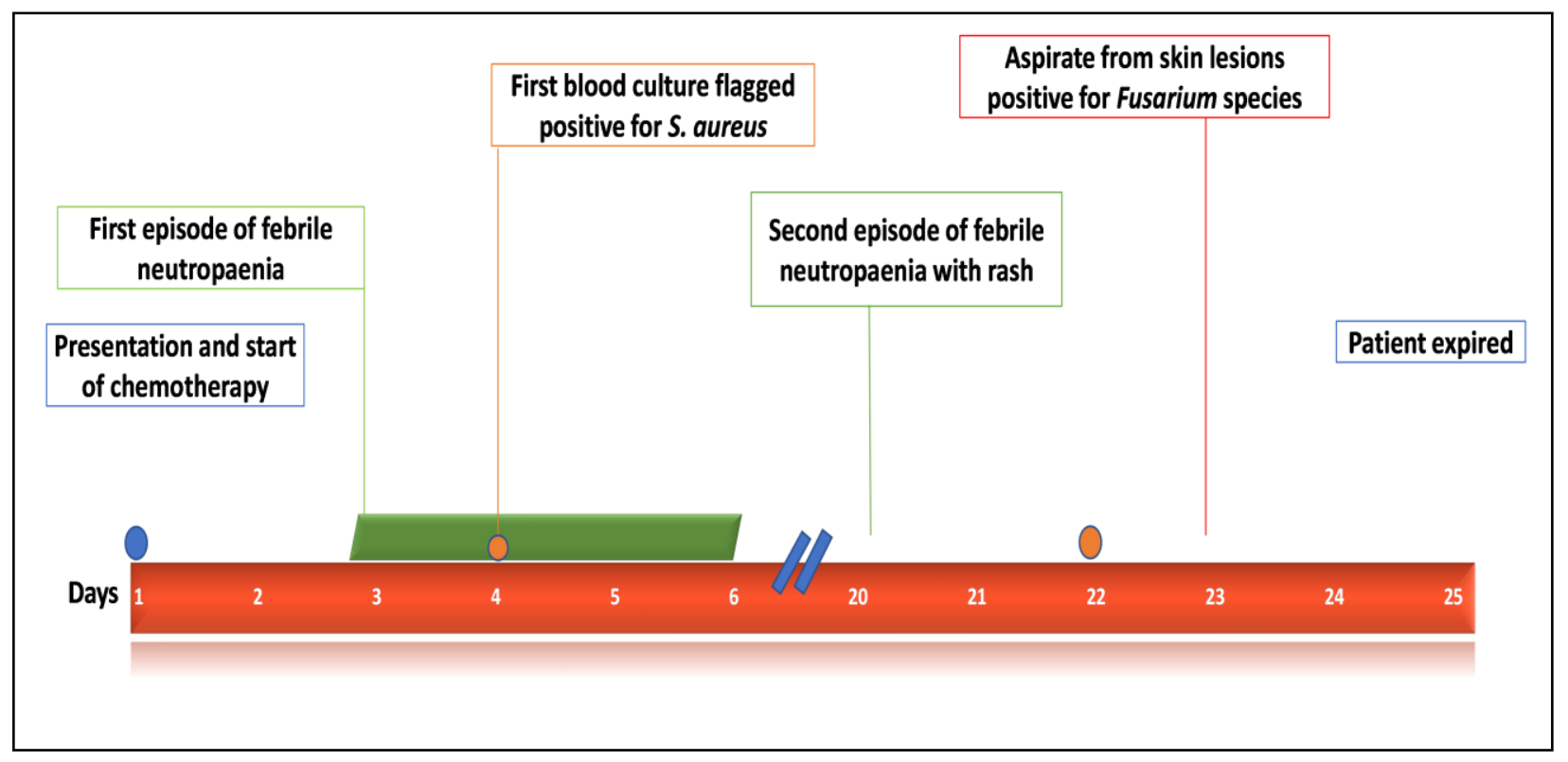

\section{Patient 2}

A 5 1/2 year old boy presented with relapsed acute lymphocytic leukaemia six months after completing chemotherapy. On admission he was asymptomatic, but neutropaenic (ANC count $630 / \mu \mathrm{L}$ ). He was started on IV vancomycin $15 \mathrm{mg} / \mathrm{kg}$ 6hourly and IV ciprofloxacin $10 \mathrm{mg} / \mathrm{kg}$ 8hourly. After 11 days, he became febrile, and IV meropenem $20 \mathrm{mg} / \mathrm{kg}$ 8hourly and IV fluconazole $12 \mathrm{mg} / \mathrm{kg}$ daily were added to this regime. The patient's fever did not subside and after 2 days he developed multiple, firm, painful subcutaneous nodules about $2 \mathrm{~cm}$ in diameter, mainly on his limbs. The patient was admitted to the Pediatric Intensive Care Unit for further management, 
where the antibiotics were changed to IV piperacillin-tazobactam $112.5 \mathrm{mg} / \mathrm{kg}$ IV every 6hours and IV amphotericin B deoxycholate $1 \mathrm{mg} / \mathrm{kg}$ daily empirically after taking blood for bacterial and fungal cultures.

Both his fungal and
bacterial automated
blood cultures
(BACTEC
signaled positive and
the direct gram stain
showed branching
fungal filaments. This
was later identified as
a Fusarium species by
the Mycology

Reference Laboratory (Figure 3). However, further speciation was difficult. Antifungal sensitivity testing was unavailable at the time.

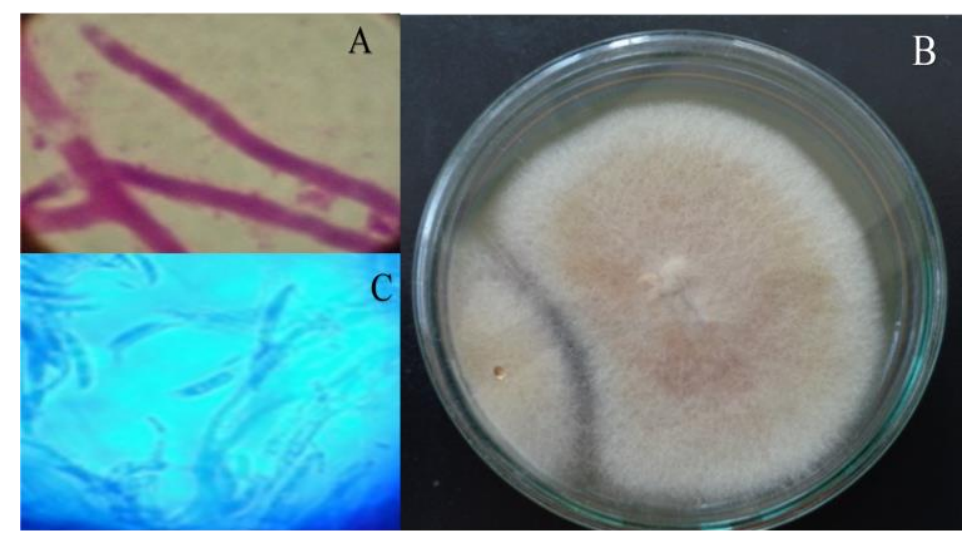

Figure 3:

A: Gram stained smear from the blood culture showing septate, branching hyphae

B: Appearance of colony on SDA one week after incubation at $26^{\circ} \mathrm{C}$ showing salmon-orange tinged floccose white colonies with orange slime

$\mathrm{C}$ : LPCB mount from the teased colony showing abundant macro-conidia with 1-3 septae and absent micro-conidia and chlamydospores

IV amphotericin B (deoxycholate) was started empirically for fever and subcutaneous nodules. Oral voriconazole $9 \mathrm{mg} / \mathrm{kg}$ 12hourly was added due to initial poor response and positive repeat blood cultures. (IV voriconazole was not available at the time.)
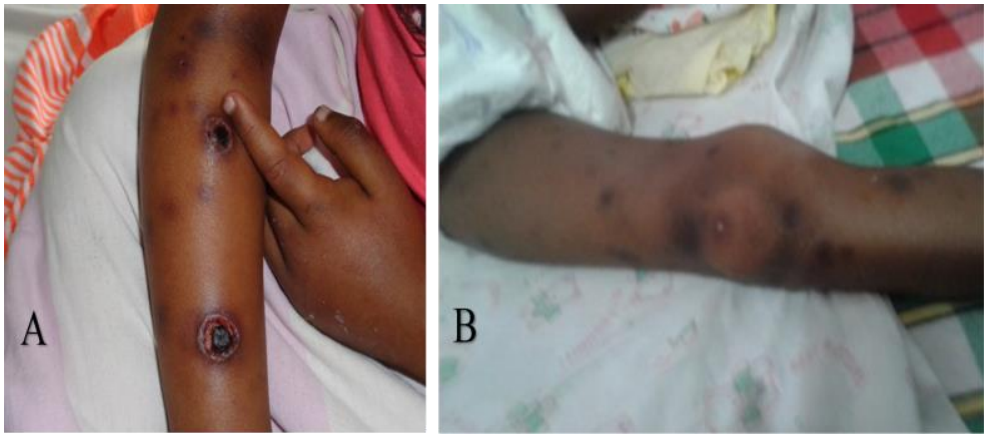

Figure 4:

A; Ulcerated subcutaneous nodules one week after onset

B: Healed lesions after completion of therapy
The patient's subcutaneous nodules became ulcerated with a necrotic center (Figure 4). Though a skin biopsy for histopathology and fungal culture were suggested, it was not carried out as the patient was at high risk of bleeding due to his low platelet count. The patient was treated with oral voriconazole and IV amphotericin B (deoxycholate) for two weeks after the first negative blood culture. $\mathrm{He}$

recovered from neutropenia and fungaemia and was discharged with healed cutaneous lesions 


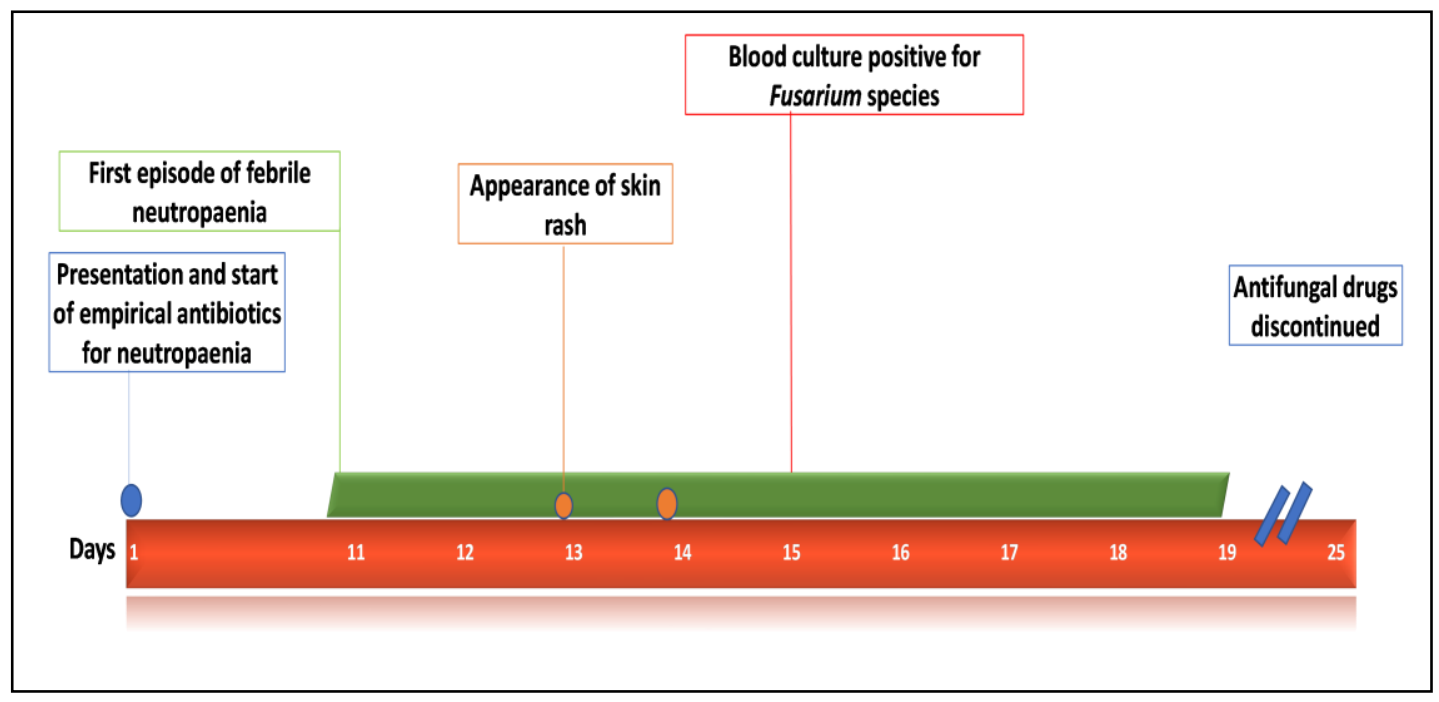

\section{Discussion}

Febrile neutropaenia occurs as a common complication of cancer chemotherapy. Although bacterial infections are the common cause of the fever, fungal infections are increasing. Those with prolonged (lasting more than seven days) and profound (ANC < 100//uL) neutropaenia are at greatest risk for invasive fungal infections. Although candidiasis and aspergillosis are by far the commonest aetiologies, other fungi are also capable of causing life-threatening infections with a variety of dermatological manifestations. ${ }^{2}$

The portal of entry for Fusarium species can be through breaches in the skin, indwelling catheters, the sinopulmonary route or the gastrointestinal tract. ${ }^{3}$ Unlike other opportunistic fungal infections, fusariosis causes frequent dermatological involvement. ${ }^{4}$ Disseminated fusariosis is mainly seen in neutropaenic patients, patients with haematological malignancies, graft-versus-host-disease and haemopoietic stem cell transplant recipients. Studies have found that in these patients, most skin manifestations $(88 \%)$ reflect disseminated infection..$^{5}$

Skin manifestations in these patients vary from multiple painful, erythematous to violaceous macules, papules or nodules. Some may resemble target lesions or ecthyma gangrenosum like lesions. Bullae have also been reported. The lesions are most often seen on the extremities. ${ }^{4,6}$ Skin biopsies and blood cultures frequently yield the organism. It is important to arrive at a diagnosis, as delays in treatment can lead to high mortality. ${ }^{4}$

The optimum therapy for disseminated fusariosis is unclear. Antifungals used in these patients included formulations of amphotericin B and the triazoles - voriconazole, posaconazole and isavuconazole, as well as a combination of a lipid formulation of amphotericin $\mathrm{B}$ and voriconazole. Reducing the immune suppressive state of the patient is also important. Hence, the use of adjunctive immunotherapy such as granulocyte or granulocyte-macrophage colonystimulating factors (G-CSF or GM-CSF) should also be considered. High mortality is seen in those 
who continue to be persistently immunosuppressed. Source control such as surgical debridement of infected tissues and the removal of infected catheters should be undertaken where feasible. ${ }^{7}$

It is recommended that those who are likely to become severely immunosuppressed, undergo a thorough skin evaluation before therapy, and that the suspicious lesions be cultured and managed accordingly. ${ }^{4}$

\section{Conclusion}

Invasive and disseminated fusariosis has high mortality, especially in immunosuppressed patients. Fungal infections should be excluded in those with febrile neutropaenia, skin manifestations and immunosuppression. Every effort should be taken to arrive at a mycological diagnosis and aggressive antifungal therapy should be initiated.

Conflicts of interest: Authors declare that there are no conflicts of interest.

Ethics Statement: Consent for publication was obtained from the patients' guardians.

\section{References}

1. CLSI. Epidemiological Cutoff Values for Antifungal Susceptibility Testing. 2nd ed. CLSI supplement M59. Wayne, PA: Clinical and Laboratory Standards Institute, 2018

2. Maddy, AJ, Sanchez, N, Shukla, BS, Maderal, AD. Dermatological manifestations of fungal infection in patients with febrile neutropaenia: A review of the literature. Mycoses. 2019; 62:826- 834. doi: https://doi.org/10.1111/myc.12928

3. Ho DY, Lee JD, Rosso F, Montoya JG. Treating disseminated fusariosis: amphotericin B, voriconazole or both? Mycoses 2007; 50:227-231. doi:10.1111/j.0933-7407.2006. 01346.x

4. Nucci M, Anaissie E. Cutaneous infection by Fusarium species in healthy and immunocompromised hosts: Implications for diagnosis and management. Clinical Infectious Diseases 2002; 35:909-20. doi: https://doi.org/10.1086/342328

5. Stempel JM, Hammond SP, Sutton DA, et al. Invasive fusariosis in the voriconazole era: singlecenter 13-year experience. Open Forum Infect Dis. 2015; 2(3):ofv099. doi:10.1093/ofid/ofv099

6. Jensen T. G., Gahrn-Hansen B., Arendrup M., Bruun B. Fusarium fungaemia in immunocompromised patients. Clin Microbiol Infect 2004; 10: 499-501. doi: https://doi.org/10.1111/j.1469-0691.2004.00859.x

7. Nucci M, Anaissie E. (2020) Treatment and prevention of Fusarium infection. In: UpToDate, Bond S. (Ed). UpTodate, Waltham, MA. Available at https://www.uptodate.com/contents/treatment-and-prevention-of-fusarium-infection. (Accessed on 17/09/2020) 